\title{
Synthesis and evaluation of novel 2-[(1,2,4-triazol-3-yl)thio] acetamide derivatives as potential serum paraoxonase-1 (PON1) activators
}

\author{
Leyla YURTTAŞ, Kaan KÜÇÜKOĞLU, Hayrünnisa NADAROĞLU, Zafer Asım KAPLANCIKLI
}

\begin{abstract}
Coronary artery disease and low-density lipoprotein (LDL) levels in the blood have long been known to be associated with peripheral vascular diseases. Paraoxonase-1 (PON1) enzyme is related to serum levels of high-density lipoprotein (HDL) and protects LDL from oxidation which may result in development of microvascular disease in diabetes. The enzyme has a major role in the prevention of atherosclerosis besides antioxidant properties. Additionally, PON1 is important in the detoxification of organophosphate insecticides from the body. In this study, we aimed to synthesize highly active new compounds which can be a drug candidate and evaluate their effects on PON1 activity.

Nine novel triazole compounds bearing thioacetamide moiety
\end{abstract}

(5a-i) were synthesized and their in vitro PON1 activity was investigated. The PON1 enzyme was purified from human serum using ammonium sulfate precipitation method. Also, it was further purified using Sepharose 4B-L-tyrosine1-naphthylamine affinity chromatography. Among the synthesized triazole compounds, $\mathbf{5 b}, \mathbf{5 c}, \mathbf{5} \mathbf{f}$ and $\mathbf{5 h}$ have been determined to increase PON1 activity, remarkably. Compounds $\mathbf{5 b}, \mathbf{5 c}, \mathbf{5 f}$ and $\mathbf{5 h}$ bearing 5-nitrothiazole, benzothiazole, 6-ethoxybenzothiazole and 6-florobenzothiazole moieties could be considered to proceed in vivo investigations which is a further stage for a drug candidate.

Key words: Triazole; thioacetamide; paraoxonase (PON1); oxidative stress; antioxidant defence
Leyla Yurttaş, Zafer Asım Kaplancıklı

Department of Pharmaceutical Chemistry, Faculty of Pharmacy, Anadolu University, Eskisehir, Turkey

Kaan Küçükoğlu

Department of Pharmaceutical Chemistry, Faculty of Pharmacy, Bülent Ecevit University, Zonguldak, Turkey

Hayrünnisa Nadaroğlu

Department of Food Technology, Erzurum Vocational Training School, Ataturk University, 25240 Erzurum, Turkey

Corresponding Author:

Leyla Yurttaş

e-mail:lyurttas@anadolu.edu.tr

Submitted / Gönderilme: 08.05.2017 Revised / Düzeltme: 20.06.2017 Accepted / Kabul: $\quad 23.06 .2017$

How to cite this article : Yurttaş L, Küçükoğlu K, Nadaroğlu H, Kaplancıklı ZA. Synthesis and evaluation of novel 2-[(1,2,4-triazol-3-yl)thio]acetamide derivatives as potential serum paraoxonase-1 (PON1) activators. Marmara Pharm J 2017; 21 (4): 967-977

\section{Introduction}

Human PON1 enzyme have been reported to comprise three enzymatic activity; phosphotriesterase, arylesterase and lactonase $[1,2]$. PON1 enzyme plays an important role to prevent atherosclerosis and its antiatherogenic properties is associated with the protection of LDL, HDL and macrophages against oxidative stress [3-5]. It prevents accumulation of oxidized lipid, LDL and therefore the development of vascular disease is avoided [6]. Low levels of PON1 enzyme is a risk factor for vascular disease, due to these roles in lipid metabolism $[7,8]$. PON1 name comes from paraoxon which is the toxic metabolite of organophosphate parathion, an insecticide. The enzyme hydrolyses various toxic oxon metabolite of the insecticide such as chlorpyrifosoxon, diazoxon as well as the nerve agents sarin, soman and VX etc. [9-13]. Phenyl acetate, thiophenyl acetate, 2-naphthyl acetate and other aromatic esters are the other PON1 substrates [14]. It is also determined to hydrolyze aromatic/aliphatic lactones and cyclic carbonates $[15,16]$. PON1 enzyme is capable of hydrolysing at least 30 kinds of lactone consisting of 4-7 atoms including drugs and endogenous compounds. It 
has more affinity for aromatic lactones. However, coumarin compound bearing $\alpha$ and $\beta$-double bond on the lactone ring can not be hydrolyzed by the PON1, but dihydrocoumarin can be hydrolysed by the enzyme $[17,18]$.

The effects of anticholesterolemic drugs statins and fibrates on the expression and activation of PON enzyme were tested and these studies have given conflicting results [19-21]. Experimental animal studies support the clinical evidence of the stimulatory effects of lipid-lowering activity of drug therapy [22]. Fibrate group of drugs gemfibrozil, fenofibrate and ciprofibrate (Figure 1) were found to increase the serum PON activity [23-26]. In patients with coronary disease, lowdose aspirin increases $\sim 13 \%$ of serum PON1 activity [27], but in healthy volunteers it was found not to increase the activity [28]. Nitro-aspirin and aspirin metabolite salicylic acid also determined to increase PON1 expression and activity [29]. Additionally, anticholesterolemic probucol and cholesterol, drug ezetimibe (Figure 1) reported to increase PON1 activity [30]. Moreover, a PPAR- $\gamma$ agonist rosiglitazone, which is used in tip 2 diabetes theraphy, have caused increase of serum PON1 activity (9-13\%) in diabetic patients [31], furthermore it increases the serum PON1 activity by increasing the synthesis of HDL particles in rabbit [32] (Figure 1).

Triazole ring is a stable, highly studied scaffold which exists in clinically used drugs and a lot of biactive molecules. Triazole derivatives have also capacity of hydrogen bonding which provides binding to biomolecular structures $[33,34]$. On the other hand, thiazoles/benzothiazoles are another important class of heterocylic compounds found in many bioactive molecules and they have been reported in a large number of literature with a wide scale of biological activities $[35,36]$.

Nowadays, studies on the PON1 enzyme are more popular around the world. Despite this situation, there are a few studies in the literature on the interaction between drugs and PON enzyme activity. Therefore, we focused on novel 1,2,4-triazole and thiazole/benzothiazole bearing thioacetamide derivatives (5a-i) which possess high efficiency on various biological application area. The scope of this study includes synthesis of nine compounds and evaluation of their PON1 increasing effects which is a preliminary stage for a drug candidate.

\section{Results}

The synthesis of title compounds was carried out according to the previous study [37] of us as shown in Figure 2. At the beginning, starting material 3-cyclohexanepropionic acid was esterificated with ethanol to give ethyl3-cyclohexylpropanoate (1). Then the obtained ester was reacted with hydrazine hydrate to give 3-cyclohexylpropanohydrazide (2). 1-(3-Cyclohexylpropionyl)-4-phenyl-3-thiosemicarbazide (3) was obtained with the reaction of hydrazide compound (2) and phenylisothiocyanate. Ring closuring product 4-phenyl-5-(3-cyclohexylpropionyl)-2,4-dihydro-3H1,2,4-triazol-3-thione (4) was synthesized by refluxing compound 3 with aqueous $\mathrm{KOH}$ solution. Finally 4-phenyl-5-(3-cyclohexylpropionyl)-3-[N-(2-thiazolyl/

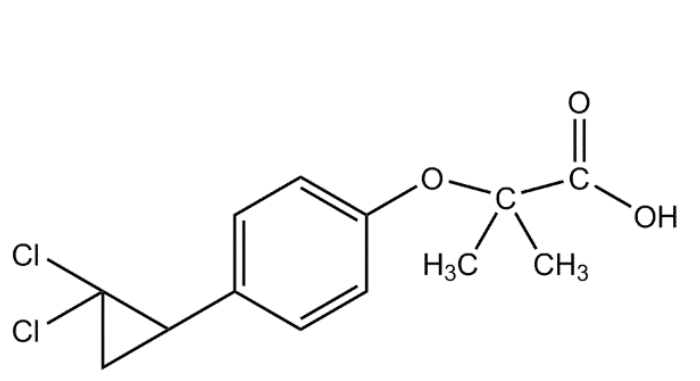

Ciprofibrate<smiles>O=C1C(CCC(O)c2ccc(F)cc2)C(c2ccc(O)cc2)N1c1ccc(F)cc1</smiles>

Ezetimibe<smiles>CN(CCOc1ccc(CC2SC(=O)NC2=O)cc1)c1ccccn1</smiles>

Rosiglitazone

Figure 1. Some PON1 enzyme activator compounds 
Table 1. Summary of Purification for PON1 from Human Serum

\begin{tabular}{|c|c|c|c|c|c|c|c|}
\hline $\begin{array}{c}\text { Purification } \\
\text { steps }\end{array}$ & $\begin{array}{c}\text { Volume } \\
(\mathrm{mL})\end{array}$ & $\begin{array}{l}\text { Activity } \\
(\mathrm{U} / \mathrm{mL})\end{array}$ & $\begin{array}{c}\text { Total Activity } \\
\text { U }\end{array}$ & $\%$ & $\begin{array}{l}\text { Protein } \\
(\mathrm{mg} / \mathrm{mL})\end{array}$ & $\begin{array}{l}\text { Spesific } \\
\text { Activity } \\
\text { (U/mg) }\end{array}$ & $\begin{array}{l}\text { Purification } \\
\quad \text { Fold }\end{array}$ \\
\hline Serum & 25 & 122.8 & 3070 & 100 & 162.5 & 1.76 & - \\
\hline $\begin{array}{l}\text { Ammonium sulphate } \\
\text { precipitation }\end{array}$ & 20 & 95.2 & 1904 & 62.0 & 42.1 & 2.26 & 1.3 \\
\hline $\begin{array}{l}\text { DEAE-Sephadex A } 50 \\
\text { Ion Exchange }\end{array}$ & 20 & 85.6 & 1712 & 55.8 & 1.15 & 74.43 & 42.3 \\
\hline \multirow[t]{2}{*}{$\begin{array}{c}\text { Purification } \\
\text { steps }\end{array}$} & & & Total Activity & & Protein & $\begin{array}{l}\text { Spesific } \\
\text { Activity }\end{array}$ & $\begin{array}{l}\text { Purification } \\
\text { Fold }\end{array}$ \\
\hline & $(\mathrm{mL})$ & $(\mathrm{U} / \mathrm{mL})$ & $\mathrm{U}$ & $\%$ & $(\mathrm{mg} / \mathrm{mL})$ & $(\mathrm{U} / \mathrm{mg})$ & \\
\hline Enzyme fraction & 50 & 125.2 & 6260 & - & 145.2 & 0.86 & - \\
\hline $\begin{array}{c}\text { Sepharose 4B-L- } \\
\text { tyrosine-1- } \\
\text { naphthylamine affinity } \\
\text { chromatograph }\end{array}$ & 20 & 61.6 & 1712 & 49.2 & 0.083 & 742.17 & 862.99 \\
\hline
\end{tabular}

benzothiazolyl)acetamido] thio-4H-1,2,4-triazole derivatives (5a-i) were synthesized by the reaction of compound 4 and some appropriate 2-chloro- $N$-(substituted thiazolyl/ benzothiazolyl)acetamide derivatives. The final compounds were yielded with a range of 75-88 \%.

Structure elucidations of the final compounds were performed with IR, ${ }^{1} \mathrm{H}-\mathrm{NMR},{ }^{13} \mathrm{C}-\mathrm{NMR}$ and $\mathrm{MS}\left(\mathrm{ES}^{+}\right)$spectroscopic methods. Characteristic stretching absorption bands belonging to $\mathrm{C}=\mathrm{O}$ groups and $\mathrm{N}-\mathrm{H}$ groups were observed at $1679-1694 \mathrm{~cm}^{-1}$ and at $3276-3293 \mathrm{~cm}^{-1}$, respectively. In the ${ }^{1} \mathrm{H}-\mathrm{NMR}$ spectra of the compounds, cyclohexyl protons were observed at about $0.84-1.68$ ppm as the furthest upfield resonances and methylene protons belonging to alkyl group were seen at about 2.46-2.56 ppm as multiplet peaks. The other methylene protons bonded to $S$ atom were observed at 4.03-4.18 ppm and $\mathrm{N}-\mathrm{H}$ protons belonging to amide moiety were observed at about 12.38-12.72 ppm as a broad singlet. In addition, aromatic protons were seen at expected regions in the spectrums. In the ${ }^{13} \mathrm{C}$-NMR spectra of the compounds, peaks at about 160-165 ppm were assigned corresponding to $-\mathrm{C}=\mathrm{O}$ carbon atom. In the mass spectra of the compounds, $\mathrm{M}+1$ peaks agreed well with the calculated molecular weight of the target compounds. All compounds gave satisfactory elemental analysis results.

PON 1 enzyme was purified from human serum using ammonium sulfate precipitation (60-80\%), DEAE-Sephadex anion exchange chromatography. PON1 enzyme was also purified from human serum using Sepharose 4B-L-tyrosine1-naphthylamine affinity chromatography. Specific activities were calculated in both serum and purified PON 1 enzyme. Paraoxon was used as a substrate for determining PON1 enzyme activity.

The result of the purification of PON1 enzyme was presented in Table 1. The ammonium sulfate precipitation method was used as first step for the enzyme purification. PON1 enzyme was obtained from human serum with a yield of $62 \%$ by ammonium sulfate fractionation and it was purificated 1.3 fold. The sample which was applied ammonium sulfate fractionation which was then applied to DEAE-Sephadex anion exchange chromatography was obtained 42.3 fold, $55.8 \%$ yield and it was purificated $74.43 \mathrm{U} / \mathrm{mg}$ protein specific activity from human PON1 enzyme (Table 1). As summarized in Table 1, the purification of PON 1 was also carried out in simple one step using Sepharose 4B-L-tyrosine1-naphthylamine affinity chromatography. The PON 1 enzyme was purified 862.99-fold with a specific activity of 742.2 (EU/mg) and 49.2\% recovery. PON 1 enzyme purified by affinity chromatography was used for further kinetic studies. The mass and purity of the enzyme was assessed with SDS-PAGE electrophoresis and a single band at $45 \mathrm{kDa}$ level was observed (Figure 3).

Compounds 5a-i were tested to determine PON1 enzyme activity in in vitro conditions. The results were represented as graphics against to compound concentration (mM) (Figure 4). The effects of the triazole compounds (5a-i) on PON1 enzyme activity were studied at three concentrations $(0.05,0.1$ ve $0.2 \mathrm{mM})$. In Figure 4 , the relative activity obtained for the control sample was taken as $100 \%$ and the 
<smiles>CC#CC1CCCC(CCC(=O)OCC)C1</smiles><smiles>CC#CCCCC(=O)NNC(=S)Nc1ccccc1</smiles><smiles>[R]NC(=O)CSc1nnc(CCC2CCCCC2)n1-c1ccccc1</smiles><smiles>[R]=COc1ccc2nc(C)sc2c1</smiles>

Figure 2. Synthesis of the compounds (5a-i). Reactants and conditions: $i$ : EtOH, $\mathrm{H}_{2} \mathrm{SO}_{4}$, reflux, $12 \mathrm{~h} ; \mathrm{ii}: \mathrm{NH}_{2} \mathrm{NH}_{2} \cdot \mathrm{H}_{2} \mathrm{O}, \mathrm{EtOH}_{\text {, }}$ reflux, 6h; iii: Phenyl isothiocyanate, reflux, 2h; iv: $2 \mathrm{~N} \mathrm{KOH}$, reflux, 3h; $v$ : 2-Chloro- $N$-(substituted thiazolyl/benzothiazolyl) acetamide derivatives, $\mathrm{K}_{2} \mathrm{CO}_{3}$, acetone, r.t, $2 \mathrm{~h}$.

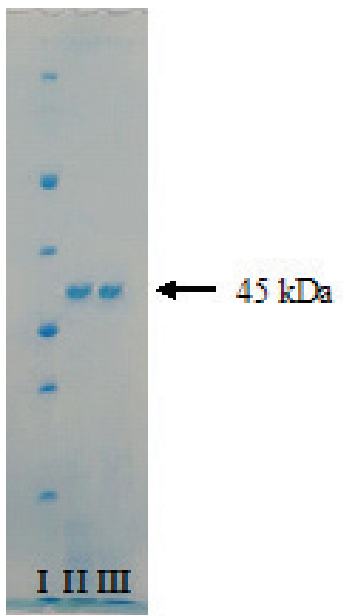

Figure 3. SDS-PAGE electrophoretic pattern of paraoxonase. [I: standart protein (myosin (200 kDa), $\beta$-galactosidase (125 $\mathrm{kDa})$, bovine serum albumin (BSA) $(66 \mathrm{kDa})$, glyceraldehyde 3-phosphate dehydrogenase (36 kDa), carbonic anhydrase (29 $\mathrm{kDa}$ ), lyzozme (14.3 kDa)); II : Purified PON 1 enzyme using ammonium sulfate precipitation (45 kDa) III: Purified PON1 enzyme using Sepharose 4B-L-tyrosine-1-naphthylamine affinity chromatography (45 kDa)]. 

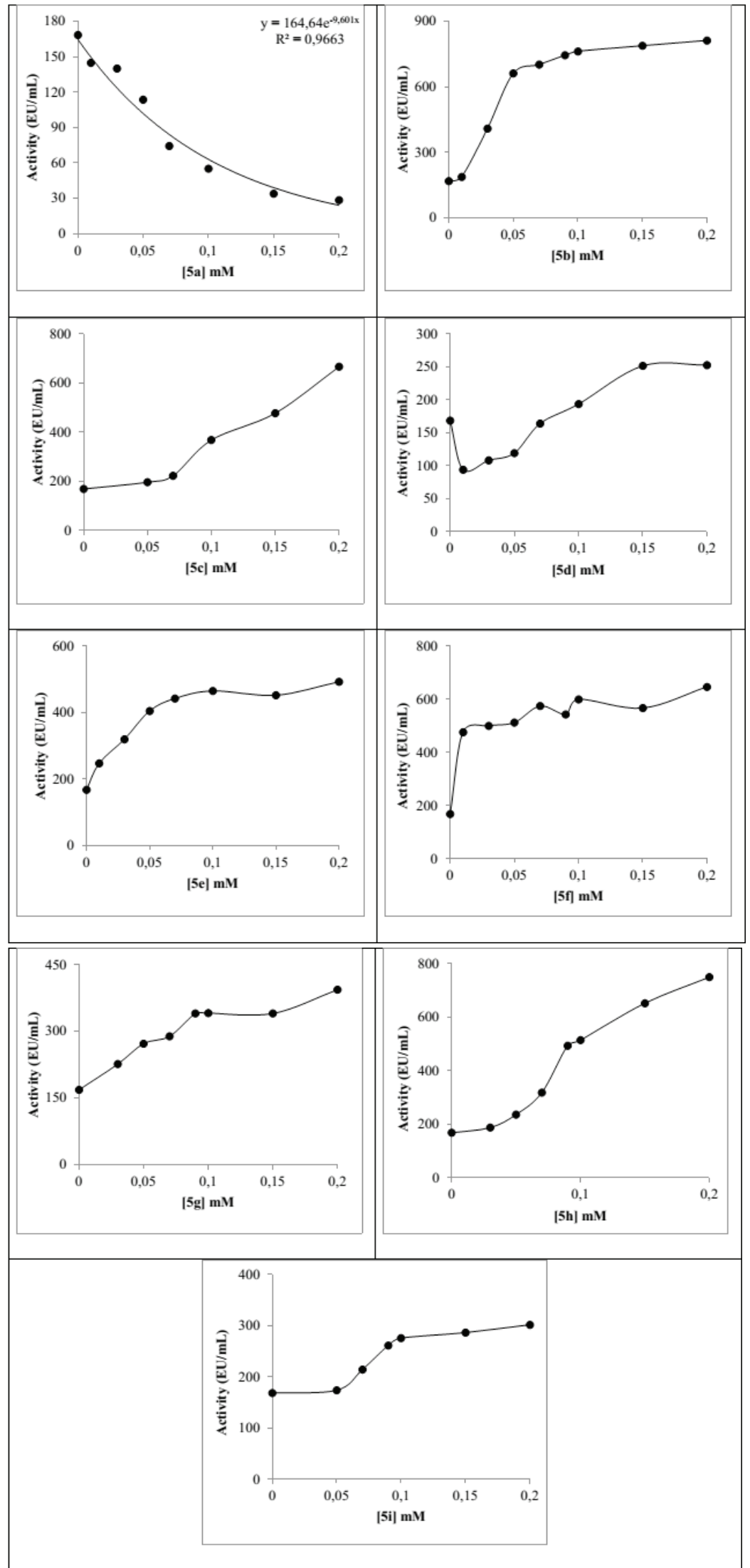

Figure 4. The in vitro PON1 enzyme activity of the compounds 5a-i. 
relative activities' values were calculated for the triazole compounds (5a-i) at indicated concentrations $(0.05,0.1$ ve $0.2 \mathrm{mM}$ ). According to the obtained data, all compounds exhibited a dose dependent increase on PON1 activity. Only compound 5a was an exception; the activator effect of this compound on PON1 decreased when the concentration increased $(67.5 \%$ activity at $0.05 \mathrm{mM} ; 32.8 \%$ activity at 0.1 $\mathrm{mM}$ concentration; $16.9 \%$ activity at $0.2 \mathrm{mM}$ concentration) (Table 2). The percentage of the relative activity of the final compounds ignored. When we compared relative percentage activity of the final compounds except $5 \mathbf{a}$, it was found $70.8 \%-392.9 \%$ at $0.05 \mathrm{mM}, 114.9 \%-452.8 \%$ at $0.1 \mathrm{mM}$ and $149.9 \%-482.5 \%$ at $0.2 \mathrm{mM}$ concentration. Compound 5b with $392.9 \%$ relative percentage and compound $\mathbf{5 f}$ with $304.5 \%$ relative percentage exhibited the highest PON1 enzyme activator activity at $0.05 \mathrm{mM}$. When the concentration was increased to $0.1 \mathrm{mM}$, compound $\mathbf{5 b}$ with $452.8 \%$ relative activity and $\mathbf{5 f}$ with $356.5 \%$ relative activity were found as the most active compounds, again. Additionally, the relative activity of the compound $\mathbf{5 h}$ was increased 2.18 fold (305.2\% relative activity), when the concentration was increased from $0.05 \mathrm{mM}$ to $0.1 \mathrm{mM}$. When the concentration was $0.2 \mathrm{mM}$, the activity was observed in sequence with $\mathbf{5 b}>\mathbf{5} \mathbf{h}>\mathbf{5} \mathbf{c}>\mathbf{5 f}$ (Relative activity percentages were determined $482.5 \%, 444.8 \%, 395.5 \%$ and $384.4 \%$, respectively). Herein, it is remarkable that the activatory effect of the compounds $\mathbf{5} \mathbf{c}$ and $\mathbf{5 h}$ were increased 3.4 and 3.17 fold, when the concentrations were changed from $0.05 \mathrm{mM}$ to $0.2 \mathrm{mM}$. As can be seen from Table 2, another important point is the increase in the activity of the compound $\mathbf{5 d}$. The relative activity of the mentioned compound was determined as $70.8 \%$ at $0.05 \mathrm{mM}$, when the concentration was incerased to $0.2 \mathrm{mM}$ the relative activity was increased to $149.9 \%$ with 2.12 fold. The \% relative activity of the other derivatives was changed less than two fold, when the concentration was increased two fold.

\section{Discussion}

The activation of the paraoxonase enzyme maintains HDL reverse cholesterol transport function by protecting it oxidation protection. This situation prevents foam cell formation by the cholesterol accumulation in the cell wall and it also prevents the development of atherosclerosis [38]. Again, as a result of the activation of PON1 enzyme, the antioxidant power of the body increases; thus it provides to treat cancer, diabetes, connective tissue diseases, hepatic, and renal pathologies such as Parkinson's disease. When the obtained results are evaluated, the synthesized triazole compounds except 5a have been activated the human PON1. Compounds $\mathbf{5 b}$ with 5 -nitrothiazole, $\mathbf{5} \mathbf{c}$ with 2-benzothiazole, 5f with 6-ethoxy-2-benzothiazole and $\mathbf{5 h}$ with 6-fluoro-2benzothiazole moieties may be considered to emerge on further studies as PON1 enzyme activator.

\section{Conclusion}

In this work, nine new triazole compounds including thiazole and benzothiazole rings were synthesized and evaluated for their increasing effects on paraoxonase-1 (PON1) activity. Compound $\mathbf{5 b}$ bearing 5-nitrothiazole moiety provoked the highest relative PON1 enzymatic activity at $0.2 \mathrm{mM}$ dose. Additionally, compounds $\mathbf{5 c}, \mathbf{5} \mathbf{f}$ and $\mathbf{5 h}$ have also been determined to increase PON1 activity, remarkably. These four compounds have come into prominence and they are thought to investigate for further in vivo studies to be a drug candidate.

Table 2. The effect of $\mathbf{5 a}-\mathbf{i}$ series on purified PON1 activity from human serum

\begin{tabular}{|c|c|c|c|c|c|c|}
\hline Compounds & $\begin{array}{c}\text { Concentration } \\
(\mathrm{mM})\end{array}$ & $\begin{array}{c}\text { Relative Activity } \\
\text { (\%) }\end{array}$ & $\begin{array}{c}\text { Concentration } \\
(\mathrm{mM})\end{array}$ & $\begin{array}{c}\text { Relative Activity } \\
\text { (\%) }\end{array}$ & $\begin{array}{c}\text { Concentration } \\
(\mathrm{mM})\end{array}$ & $\begin{array}{l}\text { Relative Activity } \\
\text { (\%) }\end{array}$ \\
\hline None & 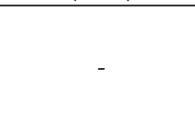 & $\begin{array}{c}100 \pm 0.0 \\
(168.4 \pm 1.1 \\
\mathrm{U} / \mathrm{mL})\end{array}$ & 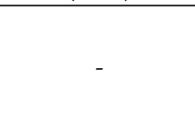 & $100 \pm 0.0$ & - & $\begin{array}{c}100 \pm 0.0 \\
(168.4 \pm 1.1 \\
\mathrm{U} / \mathrm{mL})\end{array}$ \\
\hline $5 a$ & 0.05 & 67.5 & 0.1 & 32.8 & 0.2 & 16.9 \\
\hline $5 b$ & 0.05 & 392.9 & 0.1 & 452.8 & 0.2 & 482.5 \\
\hline $5 c$ & 0.05 & 116.2 & 0.1 & 218.2 & 0.2 & 395.5 \\
\hline $5 d$ & 0.05 & 70.8 & 0.1 & 114.9 & 0.2 & 149.9 \\
\hline $5 e$ & 0.05 & 240.9 & 0.1 & 276.7 & 0.2 & 292.9 \\
\hline $5 f$ & 0.05 & 304.5 & 0.1 & 356.5 & 0.2 & 384.4 \\
\hline $5 g$ & 0.05 & 161.7 & 0.1 & 202.4 & 0.2 & 227.8 \\
\hline $5 \mathrm{~h}$ & 0.05 & 140.3 & 0.1 & 305.2 & 0.2 & 444.8 \\
\hline $5 i$ & 0.05 & 103.2 & 0.1 & 163.7 & 0.2 & 179.2 \\
\hline
\end{tabular}




\section{Materials and Methods}

\subsection{Chemistry}

All chemicals and DEAE-cellulose, glycine, $\left(\mathrm{NH}_{4}\right)_{2} \mathrm{SO}_{4}$, protein assay reagents, and chemicals for electrophoresis were purchased from Sigma-Aldrich Co. (Sigma-Aldrich Corp., St. Louis, MO, USA) and Merck Chemicals (Merck KGaA, Darmstadt, Germany). Melting points were determined using an Electrothermal 9100 digital melting point apparatus (Electrothermal, Essex, UK) and were uncorrected. Spectroscopic data were recorded on the following instruments. IR: Shimadzu 8400 FTIR spectrophotometer (Shimadzu, Tokyo, Japan); ${ }^{1} \mathrm{H}-\mathrm{NMR}$ : Bruker DPX 400 NMR spectrometer (Bruker Bioscience, Billerica, MA, USA), in DMSO- $d_{6}$, TMS as internal standard; ${ }^{13} \mathrm{C}-\mathrm{NMR}$ : Bruker DPX 100 NMR spectrometer (Bruker Bioscience, Billerica, MA, USA), in DMSO- $d_{6}$, MS: AB SCIEX-3200 Q-TRAP LC/MS/MS MASS spectrometer (Fisons Instruments Vertriebs GmbH, Mainz, Germany). Elemental analyses were performed on a Leco TruSpec Micro CHN/CHNS elemental analyzer (Leco, Michigan, USA).

5.1.1. Synthesis of ethyl 3-cyclohexylpropanoate (1). 3Cyclohexanepropionic acid $(0.20 \mathrm{~mol})$ was refluxed in excess ethanol $(0.25 \mathrm{~mol})$ for $12 \mathrm{~h}$. The obtained ester was treated with water and saline. Compound ethyl 3-cyclohexylpropanoate (1) was given with separation funnel. Boiling point was detected $105-113{ }^{\circ} \mathrm{C}$ for this compounds in a previous literature [39].

5.1.2. Synthesis of 3-cyclohexylpropanohydrazide (2). 0.15 mole of ethyl 3-cyclohexylpropanoate (1) and $0.3 \mathrm{~mol}$ of $85 \%$ hydrazine hydrate in $200 \mathrm{~mL}$ ethanol were stirred for six hours, then the precipitate was filtered and washed with water. The melting point was identified as $90^{\circ} \mathrm{C}$ according to the reported literature [40].

5.1.3. Synthesis of 1-(3-cyclohexylethyl)-4-phenyl-3thiosemicarbazide (3). Compound $2(0.1 \mathrm{~mol})$ was refluxed with phenylisothiocyanate $(0.1 \mathrm{~mol})$ in ethanol for $2 \mathrm{~h}$ and the precipitate was filtered.

5.1.4. 4-Phenyl-5-(3-cyclohexylethyl)-2,4-dihydro-3H-1,2,4triazol-3-thione (4). Compound 4 was given by refluxing compound 3 (0.05 mol) with aqueous $\mathrm{KOH}(200 \mathrm{~mL})$ solution. After $3 \mathrm{~h}$ refluxing, the reaction mixture was cooled at room temperature and neutralized with acetic acid solution and then filtered.

5.1.5. General procedure for the synthesis of 2-[[5-(2-cyclohexylethyl)-4-phenyl-4H-1,2,4-triazol-3-yl] thio]-N-(substituted thiazol/benzothiazol-2-yl)acetamide derivatives (5a-i). A mixture of compound 4 (0.002 mol), appropriate 2-chloro- $\mathrm{N}$-(substituted thiazolyl/ benzothiazolyl)acetamide derivative $(0.002 \mathrm{~mol})$ and potassium carbonate $(0.002 \mathrm{~mol})$ in acetone was stirred for $2 \mathrm{~h}$. After evaporating the solvent, the precipitate was treated with water and then filtered. Afterwards, the raw products was recrstallised from ethanol.

5.1.5.1. 2-[[5-(2-Cyclohexylethyl)-4-phenyl-4H-1,2,4-triazol3-yl]thio]-N-(thiazol-2-yl)acetamide (5a). Yield 83 \%, m.p. $207^{\circ} \mathrm{C}$. IR $\left(\mathrm{KBr}, \mathrm{cm}^{-1}\right): v_{\max } 3291$ (amide N-H), $1694(\mathrm{C}=\mathrm{O})$, 1543-1426 (C=C, C=N), 1268-1136 (C-O, C-N). ${ }^{1} \mathrm{H}-\mathrm{NMR}$ $\left(400 \mathrm{MHz}, \mathrm{DMSO}-d_{6}, \mathrm{ppm}\right): \delta 0.85-0.89(\mathrm{~m}, 2 \mathrm{H}$, cyclohexyl protons), 1.35-1.62 ( $\mathrm{m}, 9 \mathrm{H}$, cyclohexyl protons), 2.48-2.54 (m, $\left.4 \mathrm{H},-\mathrm{CH}_{2}-\mathrm{CH}_{2}-\right), 4.13\left(\mathrm{~s}, 2 \mathrm{H},-\mathrm{CO}-\mathrm{CH}_{2}\right), 7.22$ (d, J=3.6 $\mathrm{Hz}, 1 \mathrm{H}, \mathrm{Ar}-\mathrm{H}$ ), 7.43-7.47 (m, 3H, Ar-H), 7.58-7.56 (m, 3H, Ar-H), 12.38 (brs, $1 \mathrm{H}, \mathrm{N}-\mathrm{H}$ ). For $\mathrm{C}_{21} \mathrm{H}_{25} \mathrm{~N}_{5} \mathrm{OS}_{2}$ calculated: $58.99 \%$ C, $5.89 \% \mathrm{H}, 16.38 \% \mathrm{~N}, 15.00 \% \mathrm{~S}$; found: $58.94 \% \mathrm{C}$, $5.87 \% \mathrm{H}, 16.33 \% \mathrm{~N}, 15.03 \% \mathrm{~S}$. MS [M+1] $]^{+}$: m/z 428.

5.1.5.2. 2-[[5-(2-Cyclohexylethyl)-4-phenyl-4H-1,2,4-triazol3-yl]thio]-N-(5-nitrothiazol-2-yl)acetamide (5b). Yield 85 \%, m.p. $116-117^{\circ} \mathrm{C}$. IR $\left(\mathrm{KBr}, \mathrm{cm}^{-1}\right): v_{\max } 3279$ (amide N-H), $1687(\mathrm{C}=\mathrm{O}), 1564-1456\left(\mathrm{C}=\mathrm{C}, \mathrm{C}=\mathrm{N}, \mathrm{NO}_{2}\right), 1238-1152(\mathrm{C}-$ O, C-N). ${ }^{1} \mathrm{H}-\mathrm{NMR}\left(400 \mathrm{MHz}, \mathrm{DMSO}-d_{6}, \mathrm{ppm}\right): \delta 0.84-0.90$ (m, 2H, cyclohexyl protons), 1.33-1.63 (m, 9H, cyclohexyl protons), $2.48-2.53\left(\mathrm{~m}, 4 \mathrm{H},-\mathrm{CH}_{2}-\mathrm{CH}_{2}^{-}\right), 4.03(\mathrm{~s}, 2 \mathrm{H}$, - $\left.\mathrm{CO}-\mathrm{CH}_{2}\right), 7.44-7.46$ (m, 2H, Ar-H), 7.56-7.59 (m, 3H, Ar$\mathrm{H}), 8.41(\mathrm{~s}, 1 \mathrm{H}$, thiazole-H), 12.65 (brs, $1 \mathrm{H}, \mathrm{N}-\mathrm{H}) .{ }^{13} \mathrm{C}-\mathrm{NMR}$ $\left(100 \mathrm{MHz}, \mathrm{DMSO}-d_{6}, \mathrm{ppm}\right): \delta 24.48,25.23,32.44,33.38$, $39.43,40.28,127.99,130.58,133.95,137.30,146.45,150.81$, 156.08, 173.33, 174.86. For $\mathrm{C}_{21} \mathrm{H}_{24} \mathrm{~N}_{6} \mathrm{O}_{3} \mathrm{~S}_{2}$ calculated: $53.37 \%$ C, $5.12 \% \mathrm{H}, 17.78 \% \mathrm{~N}, 13.57 \% \mathrm{~S}$; found: $53.34 \% \mathrm{C}, 5.13 \%$ $\mathrm{H}, 17.74 \% \mathrm{~N}, 13.60 \% \mathrm{~S}$. MS [M+1]+: m/z 473 .

5.1.5.3. 2-[[5-(2-Cyclohexylethyl)-4-phenyl-4H-1,2,4-triazol3-yl]thio]-N-(benzothiazol-2-yl)acetamide (5c). Yield $75 \%$, m.p. $262-264{ }^{\circ} \mathrm{C}$. IR $\left(\mathrm{KBr}, \mathrm{cm}^{-1}\right): v_{\max } 3288($ amide $\mathrm{N}-\mathrm{H})$, $1679(\mathrm{C}=\mathrm{O}), 1512-1478(\mathrm{C}=\mathrm{C}, \mathrm{C}=\mathrm{N}), 1256-1110(\mathrm{C}-\mathrm{O}, \mathrm{C}-\mathrm{N})$. ${ }^{1} \mathrm{H}-\mathrm{NMR}\left(400 \mathrm{MHz}, \mathrm{DMSO}-d_{6}\right.$, ppm) : $\delta 0.85-0.93(\mathrm{~m}, 2 \mathrm{H}$, cyclohexyl protons), 1.34-1.68 (m, 9H, cyclohexyl protons), 2.49-2.56 (m, $\left.4 \mathrm{H},-\mathrm{CH}_{2}-\mathrm{CH}_{2}-\right), 4.22$ (s, $\left.2 \mathrm{H},-\mathrm{CO}-\mathrm{CH}_{2}\right), 7.30-$ 7.63 (m, 7H, Ar-H), 7.76 (d, J=7.6 Hz, 1H, Ar-H), 7.98 (d, $J=7.6 \mathrm{~Hz}, 1 \mathrm{H}, \mathrm{Ar}-\mathrm{H}$ ), 12.65 (brs, $1 \mathrm{H}, \mathrm{N}-\mathrm{H}$ ). For $\mathrm{C}_{25} \mathrm{H}_{27} \mathrm{~N}_{5} \mathrm{OS}_{2}$ calculated: $62.86 \%$ C, $5.70 \% \mathrm{H}, 14.66 \% \mathrm{~N}, 13.43 \% \mathrm{~S}$; found: $62.84 \%$ C, $5.72 \% \mathrm{H}, 14.70 \% \mathrm{~N}, 13.45 \% \mathrm{~S}$. MS [M+1]+: m/z 478.

5.1.5.4. 2-[[5-(2-Cyclohexylethyl)-4-phenyl-4H-1,2,4-triazol3-yl]thio]-N-(6-methylbenzothiazol-2-yl)acetamide $(5 d)$. Yield $79 \%$, m.p. $285^{\circ} \mathrm{C}$. IR $\left(\mathrm{KBr}, \mathrm{cm}^{-1}\right): v_{\max } 3284$ (amide 
$\mathrm{N}-\mathrm{H}), 1686(\mathrm{C}=\mathrm{O}), 1546-1438(\mathrm{C}=\mathrm{C}, \mathrm{C}=\mathrm{N}), 1230-1139(\mathrm{C}-$ O, C-N). ${ }^{1} \mathrm{H}-\mathrm{NMR}\left(400 \mathrm{MHz}, \mathrm{DMSO}-d_{6}\right.$, ppm) : $\delta 0.85-0.93$ (m, 2H, cyclohexyl protons), 1.35-1.68 (m, 9H, cyclohexyl protons), 2.41 (s, 3H, $\left.-\mathrm{CH}_{3}\right), 2.49-2.56\left(\mathrm{~m}, 4 \mathrm{H},-\mathrm{CH}_{2}-\mathrm{CH}_{2}-\right.$ ), $4.20\left(\mathrm{~s}, 2 \mathrm{H},-\mathrm{CO}-\mathrm{CH}_{2}\right), 7.47-7.49(\mathrm{~m}, 1 \mathrm{H}, \mathrm{Ar}-\mathrm{H}), 7.58-7.65$ (m, 6H, Ar-H), 7.76 (s, 1H, Ar-H), 12.59 (brs, 1H, N-H). For $\mathrm{C}_{26} \mathrm{H}_{29} \mathrm{~N}_{5} \mathrm{OS}_{2}$ calculated: $63.51 \% \mathrm{C}, 5.95 \% \mathrm{H}, 14.24 \% \mathrm{~N}$, $13.04 \%$ S; found: $63.55 \%$ C, $5.93 \% \mathrm{H}, 14.21 \% \mathrm{~N}, 13.06 \% \mathrm{~S}$. MS [M+1]+: m/z 492 .

5.1.5.5. 2-[[5-(2-Cyclohexylethyl)-4-phenyl-4H-1,2,4-triazol3-yl]thio]-N-(6-methoxybenzothiazol-2-yl)acetamide (5e). Yield $85 \%$, m.p. $264{ }^{\circ} \mathrm{C}$. IR $\left(\mathrm{KBr}, \mathrm{cm}^{-1}\right): v_{\max } 3279$ (amide $\mathrm{N}-\mathrm{H}), 1679(\mathrm{C}=\mathrm{O}), 1505-1439(\mathrm{C}=\mathrm{C}, \mathrm{C}=\mathrm{N}), 1239-1051(\mathrm{C}-$ O, C-N). ${ }^{1} \mathrm{H}-\mathrm{NMR}\left(400 \mathrm{MHz}, \mathrm{DMSO}-d_{6}, \mathrm{ppm}\right): \delta 0.85-0.93$ (m, 2H, cyclohexyl protons), 1.34-1.68 ( $\mathrm{m}, 9 \mathrm{H}$, cyclohexyl protons), $2.49-2.56\left(\mathrm{~m}, 4 \mathrm{H},-\mathrm{CH}_{2}-\mathrm{CH}_{2}-\right), 3.81$ (s, $3 \mathrm{H}$, $\left.-\mathrm{OCH}_{3}\right), 4.19$ (s, 2H, $\left.-\mathrm{CO}-\mathrm{CH}_{2}\right), 7.03$ (dd, J=8.4, $2.4 \mathrm{~Hz}, 1 \mathrm{H}$, Ar-H), 7.47-7.66 (m, 7H, Ar-H), 12.52 (brs, 1H, N-H). For $\mathrm{C}_{26} \mathrm{H}_{29} \mathrm{~N}_{5} \mathrm{O}_{2} \mathrm{~S}_{2}$ calculated: $61.51 \% \mathrm{C}, 5.76 \% \mathrm{H}, 13.80 \% \mathrm{~N}$, $12.63 \% \mathrm{~S}$; found: $61.56 \% \mathrm{C}, 5.78 \% \mathrm{H}, 13.87 \% \mathrm{~N}, 12.66 \% \mathrm{~S}$. MS [M+1]+: m/z 508 .

5.1.5.6. 2-[[5-(2-Cyclohexylethyl)-4-phenyl-4H-1,2,4-triazol3-yl]thio]-N-(6-ethoxybenzothiazol-2-yl)acetamide

(5f).

Yield $76 \%$, m.p. $228-229^{\circ} \mathrm{C}$. IR $\left(\mathrm{KBr}, \mathrm{cm}^{-1}\right): v_{\max } 3281$ (amide N-H), $1682(\mathrm{C}=\mathrm{O}), 1538-1441(\mathrm{C}=\mathrm{C}, \mathrm{C}=\mathrm{N}), 1284-1112(\mathrm{C}-$ O, C-N). ${ }^{1} \mathrm{H}-\mathrm{NMR}\left(400 \mathrm{MHz}, \mathrm{DMSO}-d_{6}, \mathrm{ppm}\right): \delta 0.84-0.88$ (m, $2 \mathrm{H}$, cyclohexyl protons), 1.23-1.61 (m, 12H, cyclohexyl protons, $\left.\mathrm{CH}_{3}\right), 2.46-2.53\left(\mathrm{~m}, 4 \mathrm{H},-\mathrm{CH}_{2}-\mathrm{CH}_{2}-\right), 4.04(\mathrm{q}, \mathrm{J}=7$ $\left.\mathrm{Hz}, 2 \mathrm{H}, \mathrm{CH}_{2}-\mathrm{CH}_{3}\right), 4.16$ (s, $\left.2 \mathrm{H},-\mathrm{CO}-\mathrm{CH}_{2}\right), 6.98$ (dd, J=8.0, $2.8 \mathrm{~Hz}, 1 \mathrm{H}, \mathrm{Ar}-\mathrm{H}), 7.43-7.62(\mathrm{~m}, 7 \mathrm{H}, \mathrm{Ar}-\mathrm{H}), 12.43$ (brs, $1 \mathrm{H}, \mathrm{N}-\mathrm{H}) .{ }^{13} \mathrm{C}-\mathrm{NMR}\left(100 \mathrm{MHz}, \mathrm{DMSO}-d_{6}, \mathrm{ppm}\right): \delta 15.35$, $24.47,25.22$, 32.42, 33.31, 36.49, 39.41, 64.32, 106.13, 115.99, $121.88,127.92,130.63,130.71,133.48,133.71,143.23,149.69$, 156.11, 156.54, 167.52. For $\mathrm{C}_{27} \mathrm{H}_{31} \mathrm{~N}_{5} \mathrm{O}_{2} \mathrm{~S}_{2}$ calculated: $62.16 \%$ C, $5.99 \% \mathrm{H}, 13.42 \% \mathrm{~N}, 12.29 \% \mathrm{~S}$; found: $61.20 \% \mathrm{C}, 5.98 \%$ $\mathrm{H}, 13.46 \% \mathrm{~N}, 12.31 \%$ S. MS [M+1]+: m/z 522 .

5.1.5.7. 2-[[5-(2-Cyclohexylethyl)-4-phenyl-4H-1,2,4-triazol3-yl]thio]-N-(6-chlorobenzothiazol-2-yl)acetamide (5g). Yield 79 \%, m.p. 272-273 ${ }^{\circ} \mathrm{C}$. IR $\left(\mathrm{KBr}, \mathrm{cm}^{-1}\right): v_{\max } 3293$ (amide $\mathrm{N}-\mathrm{H}), 1683(\mathrm{C}=\mathrm{O}), 1512-1463(\mathrm{C}=\mathrm{C}, \mathrm{C}=\mathrm{N}), 1310-1128(\mathrm{C}-$ O, C-N). ${ }^{1} \mathrm{H}-\mathrm{NMR}\left(400 \mathrm{MHz}, \mathrm{DMSO}-d_{6}, \mathrm{ppm}\right): \delta 0.81-0.89$ (m, 2H, cyclohexyl protons), 1.30-1.64 (m, 9H, cyclohexyl protons), $2.46-2.53\left(\mathrm{~m}, 4 \mathrm{H},-\mathrm{CH}_{2}-\mathrm{CH}_{2}-\right), 4.18$ (s, $2 \mathrm{H}$, - $\mathrm{CO}-\mathrm{CH}_{2}$ ), 7.42-7.59 (m, 6H, Ar-H), $7.72(\mathrm{~d}, J=8.8 \mathrm{~Hz}, 1 \mathrm{H}$, Ar-H), 8.09 (s, 1H, Ar-H), 12.72 (brs, 1H, N-H). ${ }^{13} \mathrm{C}-\mathrm{NMR}$ $\left(100 \mathrm{MHz}, \mathrm{DMSO}-d_{6}, \mathrm{ppm}\right): \delta 24.46,25.21,32.42,33.29$, $36.43,39.58,122.15,122.51,127.19,127.91,128.36,130.65$,
$130.74,133.66,133.85,148.11,149.66,156.56,159.38,168.11$. For $\mathrm{C}_{25} \mathrm{H}_{26} \mathrm{ClN}_{5} \mathrm{OS}_{2}$ calculated: $58.64 \% \mathrm{C}, 5.12 \% \mathrm{H}, 13.68 \%$ N, $12.52 \%$ S; found: $58.66 \%$ C, $5.13 \% \mathrm{H}, 13.72 \% \mathrm{~N}, 12.54$ $\%$ S. MS $[\mathrm{M}+1]+: \mathrm{m} / \mathrm{z} 512$.

5.1.5.8. 2-[[5-(2-Cyclohexylethyl)-4-phenyl-4H-1,2,4-triazol3-yl]thio]-N-(6-fluorobenzothiazol-2-yl)acetamide (5h). Yield $88 \%$, m.p. $242-244^{\circ} \mathrm{C}$. IR $\left(\mathrm{KBr}, \mathrm{cm}^{-1}\right): v_{\max } 3287$ (amide N-H), $1687(\mathrm{C}=\mathrm{O}), 1553-1426(\mathrm{C}=\mathrm{C}, \mathrm{C}=\mathrm{N}), 1285-1113(\mathrm{C}-\mathrm{O}, \mathrm{C}-\mathrm{N})$. ${ }^{1} \mathrm{H}-\mathrm{NMR}\left(400 \mathrm{MHz}, \mathrm{DMSO}-d_{6}, \mathrm{ppm}\right): \delta 0.81-0.89(\mathrm{~m}, 2 \mathrm{H}$, cyclohexyl protons), 1.30-1.64 (m, 9H, cyclohexyl protons), 2.47-2.53 (m, $\left.4 \mathrm{H},-\mathrm{CH}_{2}-\mathrm{CH}_{2}-\right), 4.18\left(\mathrm{~s}, 2 \mathrm{H},-\mathrm{CO}-\mathrm{CH}_{2}\right), 7.26$ (t, J=8.6 Hz, $1 \mathrm{H}, \mathrm{Ar}-\mathrm{H})$, 7.44-7.60 (m, 5H, Ar-H), 7.72-7.76 (m, 1H, Ar-H), 7.86 (dd, J=8.0, 2.8 Hz, 1H, Ar-H), 12.65 (brs, $1 \mathrm{H}, \mathrm{N}-\mathrm{H}) .{ }^{13} \mathrm{C}-\mathrm{NMR}\left(100 \mathrm{MHz}, \mathrm{DMSO}-d_{6}, \mathrm{ppm}\right): \delta 24.49$, $25.22,32.43,33.31,36.42,39.41,108.75,109.02,114.85$, $115.09,122.39,122.48,127.92,130.66,130.75,133.34,133.46$, 133.68, 145.94, 149.68, 156.56, 158.18, 158.51, 160.56, 167.97 . For $\mathrm{C}_{25} \mathrm{H}_{26} \mathrm{FN}_{5} \mathrm{OS}_{2}$ MS calculated: $60.58 \%$ C, $5.29 \% \mathrm{H}, 14.13$ $\% \mathrm{~N}, 12.94 \% \mathrm{~S}$; found: $60.63 \% \mathrm{C}, 5.30 \% \mathrm{H}, 14.17 \% \mathrm{~N}, 12.97$ $\%$ S. $[\mathrm{M}+1]+: \mathrm{m} / \mathrm{z} 496$.

5.1.5.9. 2-[[5-(2-Cyclohexylethyl)-4-phenyl-4H-1,2,4-triazol3-yl]thio]-N-(6-nitrobenzothiazol-2-yl)acetamide (5i). Yield $86 \%$, m.p. $235-237^{\circ} \mathrm{C}$. IR $\left(\mathrm{KBr}, \mathrm{cm}^{-1}\right): v_{\max } 3276$ (amide $\mathrm{N}-\mathrm{H}), 1684(\mathrm{C}=\mathrm{O}), 1547-1445\left(\mathrm{C}=\mathrm{C}, \mathrm{C}=\mathrm{N}, \mathrm{NO}_{2}\right), 1281-$ 1110 (C-O, C-N). ${ }^{1} \mathrm{H}-\mathrm{NMR}$ (400 MHz, DMSO- $\left.d_{6}, \mathrm{ppm}\right)$ : $\delta$ 0.85-0.91 (m, 2H, cyclohexyl protons), 1.39-1.66 (m, 9H, cyclohexyl protons), 2.51-2.57 (m, $\left.4 \mathrm{H},-\mathrm{CH}_{2}-\mathrm{CH}_{2}-\right), 4.22$ (s, $2 \mathrm{H},-\mathrm{CO}-\mathrm{CH}_{2}$ ), 7.49-7.51 (m, $\left.2 \mathrm{H}, \mathrm{Ar}-\mathrm{H}\right), 7.60-7.63(\mathrm{~m}, 3 \mathrm{H}$, Ar-H), 7.83 (d, J=9.2 Hz, 1H, Ar-H), 8.24 (d, J=8.4 Hz, 1H, Ar-H), 8.96 (s, 1H, Ar-H), 12.72 (brs, 1H, N-H). ${ }^{13} \mathrm{C}-\mathrm{NMR}$ $\left(100 \mathrm{MHz}, \mathrm{DMSO}-d_{6}, \mathrm{ppm}\right): \delta 23.71,24.45,31.65,32.54$, $36.60,38.64,118.60,119.97,121.47,127.16,129.88,129.93$, 132.32, 132.96, 142.36, 149.19, 154.04, 155.67, 165.33, 169.08 . For $\mathrm{C}_{25} \mathrm{H}_{26} \mathrm{~N}_{6} \mathrm{O}_{3} \mathrm{~S}_{2}$ MS calculated: $57.45 \% \mathrm{C}, 5.01 \% \mathrm{H}, 16.08$ \% N, $12.27 \%$ S; found: $57.43 \%$ C, $5.03 \% \mathrm{H}, 16.11 \% \mathrm{~N}, 12.29$ $\% \mathrm{~S} .[\mathrm{M}+1]+: \mathrm{m} / \mathrm{z} 523$.

\subsection{Activity Tests}

5.2.1. Purification of Paraoxonase-I (PON1) from human serum

5.2.1.1. Ammonium sulfate precipitation. Firstly, the serum was precipitated at $60-80 \%$ saturated of ammonium sulfate. At the highest concentration, PON1 enzyme could be precipitated in the range of $60-80 \%$ ammonium sulphate saturation. For this reason, the proteins in human serum which were firstly collapsed by $60 \%$ saturation were removed. 
Then, the supernatant was brought to $80 \%$ ammonium sulphate saturation. Lastly, it was centrifuged at 15.000 $x g$ for $20 \mathrm{~min}$ and the PON1 enzyme was obtained in the precipitate. Then, precipitate was solved phosphate buffer (100 mM Na-phosphate, $\mathrm{pH}=7.0$ ). The sample was placed in dialysis membrane and it was dialyzed against of dialysis buffer ( $25 \mathrm{mM}$ Na-phosphate $\mathrm{pH}=7.0$ ) during two hours.

5.2.2. Human serum paraoxonase-I activity assay. Paraoxonase activity of the enzyme was determined at $25^{\circ} \mathrm{C}$ in the presence of $1 \mathrm{mM} \mathrm{CaCl}_{2}, 50 \mathrm{mM}$ glycine $/ \mathrm{NaOH}$ buffer $(\mathrm{pH}$ $=10.5$ ) by using paraoxon a substrate. Activity measurement is based on establishing the changes in absorption of formed $p$-nitrophenol as a result of the reaction of PON1 with paraoxon at $412 \mathrm{~nm}$. $p$-Nitrophenol the molar extinction coefficient is $\mathrm{e}=18.290 \mathrm{M}^{-1} \mathrm{~cm}^{-1}$ and an enzyme unit is number of micromole of hydrolysis paraoxon in per minute [41-43].

5.2.3. Determination of effects of compounds of 5 a-i derivatives on human serum PON1 enzyme activity in vitro. Activity assays were done to determine the effect of compounds $\mathbf{5 a - i}$ on the activity of human serum PON1 by joining compounds to the cuvette at different concentrations. All compounds were solved using DMSO. The stock solutions are diluted to create the 7 different concentrations of used $\mathbf{5 a}$-i derivatives. PON1 was measured by a spectrophotometric assay. The spectrophotometric assay based on the ability of PON1 to hydrolyse paraoxon substrate (diethyl p-nitrophenyl phosphate). Blank samples were prepared using DMSO. Results were given as Activity (U/mL)-Activator Concentration (mM) graphics. The relative activity obtained for the control sample was taken as $100 \%$ and the relative activities' values were calculated for the triazole compounds (5a-i) at indicated concentrations $(0.05,0.1$ ve $0.2 \mathrm{mM})$ using Activity (U/mL)-Activator Concentration (mM) graphics.

\subsubsection{Sepharose 4B-L-tyrosine-1-naphthylamine affinity chromatograph}

The human serum was applied to the affinity column having a structure of Sepharose 4B-L-tyrosine-1-naphthylamine and equilibrated with $25 \mathrm{mM}$ Tris/ $\mathrm{HCl}(\mathrm{pH} 8.0) / 10 \mathrm{mM} \mathrm{CaCl}_{2}$. The affinity gel was washed with the solution $25 \mathrm{mM}$ Tris/ $\mathrm{HCl}(\mathrm{pH} 8.0) / 10 \mathrm{mM} \mathrm{CaCl}_{2} / 3 \mathrm{M} \mathrm{NaCl}$. Human paraoxonase 1 was eluted with the solution $25 \mathrm{mM}$ Tris/ $\mathrm{HCl}(\mathrm{pH} 8.0) / 10$ $\mathrm{mM} \mathrm{CaCl} 2$ at $0.5 \mathrm{~mL} / \mathrm{min}$. Fractions $(3 \mathrm{~mL}$ each) were collected as $3 \mathrm{~mL}$, and those with the highest PON1 activity were combined $[42,43]$.

5.2.5. Protein determination. Protein assay was performed according to the method of Bradford for all purification steps of PON1 enzyme from human serum. This method is based on the principle which Coomassie brilliant blue G-250 is binding to proteins in medium of phosphoric acid. Formed complex shows a maximum absorbance at $595 \mathrm{~nm}$. For this purpose, a standard was prepared using serum albumin. After the determination of protein content with Bradford method in the enzyme solutions, amount of protein was calculated using the standard graph. The sensitivity of this method is between 1-100 micrograms [44].

5.2.6. SDS polyacrylamide gel electrophoresis. After PON1 was purified, purity of enzyme was checked by using 3-10\% discontinuous sodium dodecyl sulfate polyacrylamide gel (SDS-PAGE) electrophoresis according to Laemmli [45].

5.2.7. Statistical analysis. All of the tests were conducted in triplicate for determination of the PON1 activities of samples. Data were expressed as mean $+/$ - standard deviation. Statistical analyses were performed using SPSS version 10.0 software (SPSS Inc., Chicago, IL, USA), and the significant differences were determined with a $95 \%$ confidence interval $(p<0.001$ and $p<0.05)$ using Tukey's test.

\section{Authorship statement}

Author contributions: Concept - L.Y; Design - Z.A.K.; Supervision - Z.A.K.; Resource - K.K.; Materials - H.N; Data Collection and/or Processing - L.Y.; Analysis and/or Interpretation - L.Y.; Literature Search - L.Y.; Writing - L.Y.; Critical Reviews - L.Y.

\section{Conflict of interest statement}

The authors report no conflicts of interest. The authors alone are responsible for the content and writing of the paper. The authors report no conflicts of interest.

\section{References}

1. Khersonsky O, Tawfik DS. Structure-reactivity studies of serum paraoxonase PON1 suggest that its native activity is lactonase. Biochemistry 2005; 44: 6371-82.

2. Goswami B, Tayal D, Gupta N, Mallika V. Paraoxonase: A multifaceted biomolecule. Clin Chimica Acta 2009; 410:1-12.

3. Aviram M, Rosenblat M. Paraoxonases 1, 2, and 3, oxidative stress, and macrophage foam cell formation during atherosclerosis development. Free Radic Biol Med 2004; 37: 1304-16.

4. Rozenberg O, Shih DM, Aviram M. Paraoxonase 1 (PON1) attenuates macrophage oxidative status: Studies in PON1 
transfected cells and in PON1 transgenic mice. Atherosclerosis 2005; 181:9-18.

5. Kumar A. Effect of simvastatin on paraoxonase 1 (PON1) activity and oxidative stress. Asian Pac J Trop Med 2010; 3 : 310-4.

6. Mackness M, Arrol S, Durrington PN. Paraoxonase prevents accumulation of lipoperoxides in low-density-lipoprotein. FEBS Lett 1991; 286: 152-4.

7. Sinan S, Kockar F, Arslan O. Novel purification strategy for human PON1 and inhibition of the activity by cephalosporin and aminoglikozide derived antibiotics. Biochimie 2006; 88: 565-74.

8. Nadaroglu H, Demir N, Demir Y, Gungor AA. An, in vitro, study of some pesticides on the activity of human serum paraoxonase (PON1). Jordan J Chem 2011; 6: 439-51.

9. Davies HG, Richter RJ, Keifer M, Broomfield CA, Sowalla J, Furlong CE. The effect of the human serum paraoxonase polymorphism is reversed with diazoxon, soman and sarin. Nat Genet 1996; 14: 334-6.

10. Draganov DI, La Du BN. Pharmacogenetics of paraoxonases: A brief review. Naunyn Schmiedebergs Arch Pharmacol 2004; 369: 78-88.

11. Li WF, Costa LG, Richter RJ, Hagen T, Shih DM, Tward A, Lusis AJ, and Furlong CE. Catalytic efficiency determines the in vivo efficacy of PON1 for detoxifying organophosphorus compounds. Pharmacogenetics 2000; 10: 767-79.

12. Costa LG, Furlong CE, editors. Paraoxonase (PON1) in health and disease: basic and clinical aspects. Norwell, MA: Kluwer Academic Publishers; 2002.

13. Demir D, Gencer N, Arslan O. An alternative purification method for human serum paraoxonase 1 and its interactions with anabolic compounds. J Enzyme Inhib Med Chem 2016; 31: 247-52.

14. Billecke S, Draganov D, Counsell R, Stetson P, Watson C, Hsu $\mathrm{C}, \mathrm{La} \mathrm{Du}$ BN. Human serum paraoxonase (PON1) isozymes $\mathrm{Q}$ and R hydrolyze lactones and cyclic carbonate esters. Drug Metab Dispos 2000; 28: 1335-42.

15. Biggadike K, Angell RM, Burgess CM, Farrell RM, Hancock AP, Harker AJ, Irving WR, Ioannou C, Procopiou PA, Shaw RE, Solanke YE, Singh OM, Snowden MA, Stubbs RJ, Walton $\mathrm{S}$, Weston HE. Selective plasma hydrolysis of glucocorticoid gamma-lactones and cyclic carbonates by the enzyme paraoxonase: an ideal plasma inactivation mechanism. J Med Chem 2000; 43: 19-21.

16. Jakubowski H. Calcium-dependent human serum homocysteine thiolactone hydrolase. A protective mechanism against protein $N$-homocysteinylation. J Biol Chem 2000; 275 : 3957-62.

17. Garrett ER, Lippold BC and Mielck JB. Kinetics and mechanisms of lactonization of coumarinic acids and hydrolysis of coumarins I. J Pharm Sci 1971; 60: 396-405.

18. Solmaz Avcikurt A, Sinan S, Kockar F. Antidepressant and antipsychotic drugs differentially affect PON1 enzyme activity. J Enzyme Inhib Med Chem 2015; 30: 245-9.

19. Aviram M and Rosenblat M. Paraoxonases and cardiovascular diseases: pharmacological and nutritional influences. Curr Opin Lipidol 2005; 16: 393-9.
20. Camps J, Marsillach J, Joven J. Pharmacological and lifestyle factors modulating serum paraoxonase-1 activity. Mini Rev Med Chem 2009; 9: 911-20.

21. Costa LG, Giordano G, Furlong CE. Pharmacological and dietary modulators of paraoxonase 1 (PON1) activity and expression: The hunt goes on. Biochem Pharmacol 2011; 81: 337-44.

22. Zhi-Gang S, Hou-Zao C, Fei YY, Liang LH, De-Pei L. The human paraoxonase gene cluster as a target in the treatment of atherosclerosis. Antioxid Redox Signal 2012; 16: 597-632.

23. Balogh Z, Seres I, Harangi M, Kovacs P, Kakuk G, and Paragh G. Gemfibrozil increases paraoxonase activity in type 2 diabetic patients. A new hypothesis of the beneficial action of fibrates. Diabetes Metab 2001; 27: 604-10.

24. Paragh G, Seres I, Harangi M, Balogh Z, Illyes L, Boda J, Szilvassy Z, and Kovacs P. The effect of micronised fenofibrate on paraoxonase activity in patients with coronary heart disease. Diabetes Metab 2003; 29: 613-8.

25. Paragh G, Seres I, Harangi M, Erdei A, Audikovszky M, Debreczeni L, Kovacsay A, Illyes L, and Pados G. Ciprofibrate increases paraoxonase activity in patients with metabolic syndrome. Br J Clin Pharmacol 2006; 61: 694-701.

26. Yesilbursa D, Serdar A, Saltan Y, Serdar Z, Heper Y, Guclu S, and Cordan J. The effect of fenofibrate on serum paraoxonase activity and inflammatory markers in patients with combined hyperlipidemia. Kardiol Pol 2005; 62: 526-30.

27. Blatter-Garin MC, Kalix B, De Pree S, James RW. Aspirin use is associated with higher serum concentrations of the antioxidant enzyme, paraoxonase-1. Diabetologia 2003; 46: 593-4.

28. Kurban S and Mehmetoglu I. Effects of acetylsalicylic acid on serum paraoxonase activity, Ox-LDL, coenzyme Q10 and other oxidative stress markers in healthy volunteers. Clin Biochem.2010; 43: 287-90.

29. Santanam N and Parthasarathy S. Aspirin is a substrate for paraoxonase-like activity: implications in atherosclerosis. Atherosclerosis. 2007; 191: 272-5.

30. Hong SC, Zhao SP, and Wu ZH. Probucol upregulates paraoxonase 1 expression in hepatocytes of hypercholesterolemic rabbits. J Cardiovasc Pharmacol 2006; 47: 77-81.

31. van Wijk J, Coll B, Cabezas MC, Koning E, Camps J, Mackness B, Joven J. Rosiglitazone modulates fasting and post-prandial paraoxonase 1 activity in type 2 diabetic patients. Clin Exp Pharmacol Physiol 2006; 33: 1134-7.

32. Carreon-Torres E, Rendon-Sauer K, Monter-Garrido M, Toledo-Ibelles P, Gamboa R, Menjivar M, Lopez-Marure R, Luc G, Fievet C, Cruz D, Vargas-Alarcon G, and Perez-Mendez O. Rosiglitazone modifies HDL structure and increases HDLapo AI synthesis and catabolicrates. Clin Chim Acta 2009; 401: 37-41.

33. Kumar SS, Kavitha HP. Synthesis and biological applications of triazole derivatives - A review. Mini Rev Org Chem 2013; 10: 40-65.

34. Al-Masoudi IA, Al-Soud YA, Al-Salihi NJ, Al-Masoudi NA. 1,2,4-Triazoles: Synthetic approaches and pharmacological importance. (Review). Chem Het Comp 2006; 42: 1377-1403. 
35. Leoni A, Locatelli A, Morigi R, Rambaldi M. Novel thiazole derivatives: a patent review (2008 - 2012; Part 1). Expert Opin Ther Pat 2014; 24: 201-16.

36. Gill RK, Rawal RK, Bariwal J. Recent advances in the chemistry and biology of benzothiazoles. Arch Pharm Chem Life Sci 2015; 348: 155-78.

37. Turan-Zitouni G, Kaplancıklı ZA, Yıldız MT, Chevallet P, Kaya D. Synthesis and antimicrobial activity of 4-phenyl/ cyclohexyl-5-(1-phenoxyethyl)-3-[N-(2-thiazolyl)acetamido] thio-4H-1,2,4-triazole derivatives. Eur J Med Chem 2005; 40: 607-13.

38. Ekmekçi H. Paraoksonaz. Cerrahpaşa Tip Derg 2004; 35: 7882.

39. De Benneville PL, Connor R. The hydrogenation of coumarin and related compounds. J Am Chem Soc 1940; 62: 283-7.

40. Gobis K, Foks H, Bojanowski K, Augustynowicz-Kopeć E, Napiórkowska A. Synthesis of novel 3-cyclohexylpropanoic acid-derived nitrogen heterocyclic compounds and their evaluation for tuberculostatic activity. Bioorg Med Chem; 2012; 20: 137-44.

41. Renault F, Chabrière E, Andrieu J, Dublet B, Massona P, Rochua D. Tandem purification of two HDL-associated partner proteins in human plasma, paraoxonase (PON1) and phosphate binding protein (HPBP) using hydroxy apatite chromatography. J Chromatogr B 2006; 836: 15-21.

42. Demir Y, Nadaroğlu H, Demir N. Effect of glimepride on paraoxonase activity. Pharm Biol 2006; 44: 396-9.

43. Demir N, Nadaroğlu H, Demir Y. Purification of human serum paraoxonase and effect of acetyl salicylic acid on paraoxonase activity. Pharm Biol 2008; 46: 393-9.

44. Bradford MM. A rapid and sensitive method for the quantitation of microgram quantities of protein utilizing the principle of protein-dye binding. Anal Biochem 1976; 72: 248-54.

45. Laemmli UK. Cleavage of the structural proteins during the assembly of the head of bacteriophage T4. Nature1970; 227: 680-5. 